\title{
Absence d'effet de l'incorporation d'un phosphopeptide du lait sur l'utilisation du calcium et du phosphore chez le jeune porc
}

\author{
A. Pointillart et L. Guéguen \\ avec la collaboration technique de P. Camus, B. Cayron et C. Colin
}

INRA, station de recherches de nutrition, 78350 Jouy-en-Josas, France

(reçu le 19 janvier 1989, accepté le 3 juillet 1989)

Résumé - Les phosphopeptides issus de la caséine sont prétendus augmenter l'absorption intestinale du calcium. Une étude de 50 jours a été effectuée sur des porcs âgés de 6 semaines et recevant soit un régime témoin ( $T$ ), soit un régime contenant $5 \%$ d'un phosphopeptide du lait (PP). Les 2 régimes contenaient les mêmes quantités de $\mathrm{Ca}(0,8 \%)$, de $\mathrm{P}(0,5 \%)$, de protéines, d'énergie et de vitamines. Environ la moitié du calcium total, le tiers du phosphore total et le cinquième des protéines du régime PP étaient fournis par le phosphopeptide. Les excrétions fécale et urinaire, l'absorption et la rétention de $\mathrm{Ca}$ et $\mathrm{P}$ ont été mesurées après un bilan de 10 jours. Le tibia et le péroné ont été prélevés à l'abattage pour déterminer la densité, le moment de flexion et le contenu en minéraux.

Le régime alimentaire, T ou PP, n'a eu aucune influence sur l'absorption du calcium ni sur les paramètres osseux (dont l'hydroxyprolinurie). L'absorption de $\mathrm{P}$, mais pas sa rétention, était légèrement plus élevée dans le lot témoin. L'excrétion urinaire de Ca était plus forte et celle de $P$ plus faible chez les porcs recevant le phosphopeptide, ce qui pourrait provenir de différences de nature du phosphore alimentaire, plutôt que de celle des protéines.

phosphopeptide - phosphore - calcium - porc

Summary - Effect of casein phosphopeptides on calcium and phosphorus utilization in growing pigs. Casein phosphopeptides are known to influence calcium absorption. A 50-day study was performed in 6-week old pigs fed either a control diet or a $5 \%$ casein phosphopeptidecontaining diet ( $P$ P group). Both diets provided similar amounts of $\mathrm{Ca}(0.8 \%), P(0.5 \%)$, proteins, energy and vitamins. $P P$ diet provided near $1 / 2$ of total $\mathrm{Ca}, 1 / 3$ of total $P$ and $1 / 5$ of proteins in the form of casein phosphopeptide. Ca and $P$ excretion, absorption and retention were evaluated during a 10-day balance study. Bones were collected at slaughter to determine density, bending moment and bone mineral content.

Calcium absorption and bone parameters (urinary hydroxyproline included) were not influenced by the type of diet. $P$ absorption, but not retention, was slightly higher in the control group. Urinary 
Ca was higher and urinary $P$ lower in PP pigs than in controls. These changes might result from the different kinds of dietary phosphorus, inorganic versus phosphopeptide, rather than from the difference between dietary proteins.

phosphopeptide - phosphore - calcium - pig

\section{INTRODUCTION}

Les produits laitiers constituent la source principale du calcium consommé par l'homme. La majeure partie de ce calcium est liée aux protéines du lait et plus particulièrement aux caséines, jusqu'à $95 \%$ dans le lait de rate selon une étude très récente (Blake \& Henning, 1988).

Les phosphopeptides obtenus in vitro par digestion trypsique et pepsique de la caséine sont de puissants facteurs de solubilisation du calcium. Ainsi, Reeves \& Latour (1958) montrent qu'un hydrolysat, par du suc pancréatique, de caséine peut maintenir en solution le phosphate bicalcique même en ébullition prolongée. Un hydrolysat trypsique de caséine stimule le transfert de ${ }^{45} \mathrm{Ca}$ dans l'anse duodénale isolée de poulets rachitiques ou non (Mykkanen \& Wasserman, 1980). Cet effet serait attribué à la capacité des phosphopeptides à prévenir la précipitation du calcium par les phosphates dans la lumière intestinale (Naito \& Suzuki, 1974). Lee et al. (1979, 1980, 1983) montrent que la digestion de la caséine par le rat produit des phosphopeptides similaires à ceux obtenus in vitro par digestion enzymatique et que, comparée à d'autres protéines, la caséine permet une augmentation de la concentration en calcium soluble du contenu luminal. Les mêmes auteurs (Sato et al., 1983), en répétant leurs essais avec de la caséine déphosphorylée, montrent que l'effet de solubilisation calcique disparaît. Toutefois, cet effet favorable à l'absorption calcique ne semble pas inhérent au phosphore lui-même comme le suggèrent les résultats de Mykkanen et Wasserman (1980). L'équipe japonaise (Sato et al., 1986) a également observé une augmentation du transfert calcique, mesuré sur anse iléale isolée, et une meilleure rétention osseuse de ${ }^{45} \mathrm{Ca}$ avec le caséinophosphopeptide en comparaison avec un isolat protéique de soja. In vitro, l'hydrolysat trypsique de caséine permet également une augmentation de la calcification mesurée avec des cultures embryonnaires d'os de rat (Gerber \& Jost, 1986) parallèlement à un effet de solubilisation du calcium dans le milieu.

Chez le porcelet de 4 jours, la réalisation d'un court bilan (72 h) montre qu'à niveau protéique égal, les régimes contenant de la caséine conduisent à une plus grande rétention de $\mathrm{Ca}$ et $\mathrm{Mg}$ que ceux contenant des protéines de soja; le doublement de l'apport de caséine (40 vs $20 \%$ ) augmente l'absorption de $\mathrm{Ca}, \mathrm{P}$ et $\mathrm{Mg}$ tandis que le contraire est observé avec l'isolat protéique de soja (Hendricks et al., 1970). Chez le Rat, à niveau calcique égal, les régimes contenant du caséinate de calcium permettent une disponibilité du calcium (mesurée par la technique du rapport des pentes de minéralisation osseuse) supérieure à celle du carbonate (Wong \& Lacroix, 1980).

Il existe cependant plusieurs travaux très récents qui tendent à prouver que la biodisponibilité du calcium des produits laitiers n'est pas supérieure à celle d'autres formes d'apport calcique minérales ou non (acétate, chlorure, gluconate, lactate, carbonate, citrate) (Smith et al., 1987; Recker et al., 1988; Sheikh et al., 1987; Greger et al., 1987). A l'exception 
de ceux de Greger et al. (1987) estimant l'absorption calcique par la méthode des bilans sur une période d'environ un mois, les autres essais sont réalisés en temps très court, parfois moins de $24 \mathrm{~h}$. Enfin, la plupart des résultats obtenus directement avec les phosphopeptides proviennent de l'utilisation de méthodes in vitro, autrement dit de mesures indirectes de l'absorption calcique.

C'est pourquoi nous avons étudié, par comparaison avec des porcs recevant un régime classique, les effets sur le métabolisme phosphocalcique et la minéralisation osseuse de la substitution d'une partie des protéines de ce régime (essentiellement tourteau de soja) par un caséinophosphopeptide, à raison de $5 \%$, apportant pratiquement la moitié du calcium ingéré, $1 / 3$ de $P$ et $1 / 5$ des protéines. Ce phosphopeptide, riche en calcium $(7 \%)$ et en phosphore $(3 \%)$ résultait de l'hydrolyse in vitro par la trypsine de la caséine du lait. Le faible pourcentage de phosphopeptide utilisé était justifié par des considérations pratiques : il était question de voir si ce produit pouvait ou non constituer une source de calcium hautement disponible et non de comparer les effets de 2 sources de protéines, complètement différentes, sur l'utilisation du calcium.

\section{MATÉRIEL ET MÉTHODES}

\section{Animaux et régimes}

Douze porcelets Large-White pesant $13,3 \pm 0,5$ $\mathrm{kg}$, âgés de 44 jours, ont été répartis en 2 lots, 6 recevant l'aliment expérimental contenant $5 \%$ de phosphopeptide (lot PP) et 6 l'aliment témoin (lot T). Après 52 jours, les animaux furent abattus pour permettre les prélèvements osseux mais, auparavant, un bilan de 10 jétait réalisé sur 6 animaux du lot $P P$ et 5 du lot $T$, après une période d'un mois d'adaptation aux régimes et une semaine d'adaptation aux cages. La composition complète des aliments et les principales caractéristiques du phosphopeptide utilisé figurent dans le Tableau I. Les 2 aliments répondent aux besoins des porcs et assurent des apports comparables en énergie

Tableau I. Composition des régimes (\%).

$\begin{array}{lll}\text { Régimes } & T & P P\end{array}$

\begin{tabular}{|c|c|c|}
\hline Blé & 75 & 79,4 \\
\hline Tourteau de soja-50 & 22 & 14 \\
\hline Caséinophosphopeptide 1 & - & 5 \\
\hline Chlorure de sodium & - & 0,25 \\
\hline Carbonate de calcium & 2 & 1,1 \\
\hline Phosphate monosodique, $2 \mathrm{H}_{2} \mathrm{O}$ & 0,9 & - \\
\hline CMV 2 & 0,15 & 0,15 \\
\hline Calcium & 0,84 & 0,80 \\
\hline Phosphore total & 0,55 & 0,51 \\
\hline
\end{tabular}


(3 $300 \mathrm{kcal}$ d'énergie digestible $/ \mathrm{kg}$ ), en protéines (19\% de protéines brutes, aminoacides soufrés (méthionine + cystine) : $0,7 \%$; $0,3 \%$ de méthionine pour $T$ et $0,4 \%$ pour $P P$; $0,9 \%$ de lysine, en vitamines et en minéraux. Au cours du bilan, les rapports en calcium (15 $\mathrm{g} / \mathrm{j})$ et phosphore $(10 \mathrm{~g} / \mathrm{j})$ étaient pratiquement identiques à ceux recommandés par I'INRA pour les porcs en croissance de $35 \mathrm{~kg}$ (Guéguen \& Perez, 1981); le régime PP n'était pas supplémenté en phosphore minéral, tout le phosphore ayant été apporté par les matières premières : blé, tourteau de soja et phosphopeptide. Cela a nécessité l'incorporation de $0,9 \%$ de phosphate monosodique dihydraté au régime témoin pour obtenir une teneur équivalente en phosphore total. Ainsi, $33 \%$ du phosphore du régime $T$ était sous forme minérale, le phosphopeptide fournissant également un tiers du phosphore total dans le régime $\mathrm{PP}$ et les apports de phosphore végétal étant comparables pour les 2 régimes $(0,37$ et $0,33 \%$ pour T et PP respectivement). Pour le régime témoin, pratiquement la totalité (90\%) du calcium était apportée par le carbonate contre la moitié $(51 \%)$ pour le régime PP, $40 \%$ étant apportés par le phosphopeptide. Avec le régime témoin, environ la moitié $(55,5$ $\%$ ) des protéines venaient du tourteau de soja contre un tiers (35\%) avec le régime PP; ainsi les $5 \%$ de phosphopeptide apportaient environ $1 / 5$ (18\%) des protéines. On peut donc considérer qu'il y a pratiquement substitution de $5 \%$ de phosphopeptide à $7 \%$ de tourteau de soja.

\section{Prélèvements et mesures}

Le sang collecté à l'abattage a permis la mesure des concentrations plasmatiques de calcium, de phosphore inorganique et de l'activité phosphatase alcaline. Le tibia et le péroné droits ont été utilisés pour déterminer la densité apparente et la résistance à la rupture, évaluée par le moment de flexion, ainsi que la composition minérale (sur le tibia seulement : teneur en cendres, $\mathrm{Ca}$ et $\mathrm{P}$ ). Le moment de flexion $(M)$ correspond à la force nécessaire ( $F$ en newton) pour rompre une longueur ( $L, m)$ déterminée d'os, cette force étant appliquée au milieu de la diaphyse, celle-ci reposant sur deux couteaux. Ce moment de flexion est calculé suivant la formule : $M=F \times L / 4$. La mesure a été réalisée sur une machine universelle de testage (INSTRON 1102). La résorption osseuse a été estimée par dosage de l'hydroxyproline dans les urines récoltées au cours du bilan. Le bilan a permis de calculer le coefficient d'utilisation digestive apparente (CUDa, en \% de l'ingéré), le coefficient de rétention des minéraux ainsi que les excrétions, urinaire et fécale, de $\mathrm{Ca}$ et $\mathrm{P}$. La méthodologie des mesures est identique à celle précédemment décrite (Pointillart et al., 1986). Les résultats sont présentés sous forme de moyennes \pm écarts types des moyennes comparées à l'aide du test $t$ de Student.

\section{RÉSULTATS}

\section{Performances}

La vitesse de croissance, l'indice de consommation et les poids vifs à l'abattage sont comparables pour les 2 groupes de porcs (Tableau II).

\section{Calcémie, phosphatémie, phosphata- sémie}

Elles ne sont pas modifiées par la nature des régimes (Tableau III).

\section{Absorption et rétention des minéraux}

L'incorporation de $5 \%$ de phosphopeptide, malgré sa contribution pour au moins $40 \%$ du calcium consommé, n'a eu aucun effet sur les divers paramètres du bilan calcique à l'exception de la calciurie, significativement plus élevée chez les porcs du lot PP que chez les témoins 
Tableau II. Performances.

\begin{tabular}{ccc}
\hline Régimes & $T$ & $P P$ \\
\hline Poids vif à l'abattage, $\mathrm{kg}$ & $48,8 \pm 0,9$ & $49,2 \pm 1,1$ \\
Gain moyen quotidien, $\mathrm{kg}$ & $0,68 \pm 0,01$ & $0,69 \pm 0,02$ \\
Indice de consommation' & $2,32 \pm 0,10$ & $2,47 \pm 0,14$ \\
\hline
\end{tabular}

IIC : kg d'aliment consommé par kg de gain de poids, calculé durant le séjour en cages individuelles ( 3 dernières semaines).

(Tableau IV). Avec le régime PP, l'absorption du phosphore tend à être légèrement inférieure à celle observée avec le régime témoin. Cette différence est significative quand l'absorption est exprimée en g/jour, mais marque seulement une tendance quand elle est exprimée en valeur relative $(\%$ de l'ingéré). Cet écart disparaît au niveau de la rétention en raison d'une phosphaturie 3 fois plus forte chez les témoins, ce qui s'exprime clairement lorsqu'on rapporte la rétention aux quantités absorbées : la proportion absorbée qui est retenue est significativement plus importante avec le régime $\mathrm{PP}$ (Tableau IV).

\section{Paramètres osseux}

Aucun des paramètres osseux en rapport avec la minéralisation, la résistance à la rupture ou la résorption n'a été influencé par la présence du caséinophosphopeptide dans le régime (Tableau V). Ces résultats sont cohérents avec les rétentions similaires de $\mathrm{Ca}$ et $\mathrm{P}$ observées lors du bilan.

\section{DISCUSSION}

Le remplacement d'environ un tiers de lapport total de tourteau de soja par $5 \%$

Tableau III. Calcémie, phosphatémie et phosphatasémie.

\begin{tabular}{lcc}
\hline \multicolumn{1}{c}{ Régimes } & $T$ & $P P$ \\
\hline $\mathrm{Ca}, \mathrm{mg} / 100 \mathrm{ml}$ & $10,92 \pm 0,06$ & $10,53 \pm 0,21$ \\
$\mathrm{P}, \mathrm{mg} / 100 \mathrm{ml}$ & $8,27 \pm 0,14$ & $8,42 \pm 0,18$ \\
Phosphatase alcaline, Ul/1 & $147 \pm 18$ & $133 \pm 10$ \\
\hline
\end{tabular}

Aucun écart n'est significatif 
Tableau IV. Bilans de Ca et P.

Calcium

$T$
$P P$
$10,3 \pm 0,5$

$4,2 \pm 0,3$

$6,1 \pm 0,3$

$59 \pm 2$

$51 \pm 1$

$0,18 \pm 0,02$

$7,5 \pm 0,5$

$0,27 \pm 0,03^{\star \star}$

$7,5 \pm 0,3$

$48 \pm 2$

$98 \pm 0,2$
$1,23 \pm \quad 0,07$

$4,8 \pm 0,3$

$47 \pm 2$

$96 \pm 0,4^{*} \quad 79 \pm 1,7$
$9,8 \pm 0,4$

$4,5 \pm 0,3$

$5,3 \pm 0,1^{*}$

$54 \pm 2^{\circ}$

$0,36 \pm 0,05^{\star \star *}$

$5,0 \pm 0,1$

$51 \pm 2$

$93 \pm 0,9^{\star \star \star}$

Signification statistique des differences entre T et PP (test $t):{ }^{\circ} 0,05<P<0,10 ;{ }^{*} P<0,05 ;{ }^{* *} P<0,02 ;{ }^{* * *} P<0,001$.

d'un phosphopeptide provenant de l'hydrolyse in vitro de la caséine n'a pas influencé la rétention des minéraux, que celle-ci soit examinée au niveau du bilan ou au niveau osseux. En revanche, au régime contenant $5 \%$ de phosphopeptide correspondait une légère diminution de l'absorption du phosphore et une modification des excrétions urinaires de $\mathrm{Ca}$ et $\mathrm{P}$ allant dans le sens d'une hypercalciurie et d'une hypophosphaturie. Que le changement de la nature protéique

Tableau V. Paramètres osseux.

\begin{tabular}{|c|c|c|c|c|c|c|}
\hline Régimes & & $T$ & & & $P P$ & \\
\hline \multicolumn{7}{|l|}{ Densité, $\left(\mathrm{g} / \mathrm{cm}^{3}\right)$} \\
\hline Tibia & 1,19 & \pm & 0,006 & 1,18 & \pm & 0,003 \\
\hline Péroné & 1,12 & \pm & 0,01 & 1,14 & \pm & 0,006 \\
\hline \multicolumn{7}{|l|}{ Moment de flexion, Nxm } \\
\hline Tibia & 39,8 & \pm & 1,5 & 40,7 & \pm & 2,3 \\
\hline Péroné & 4,7 & \pm & 0,3 & 5,4 & \pm & 0,4 \\
\hline \multicolumn{7}{|l|}{ Contenu en minéraux des tibias } \\
\hline Cendres totales, $\mathrm{g}$ & 24,3 & \pm & 0,9 & 24,2 & \pm & 0,6 \\
\hline Cendres, $g / 100 \mathrm{~cm}^{3}$ & 27,2 & \pm & 0,7 & 26,2 & \pm & 0,5 \\
\hline Cendres, $\% \mathrm{MS}^{1}$ & 43,2 & \pm & 3,8 & 41,0 & \pm & 0,7 \\
\hline $\mathrm{Ca}, \% \mathrm{MS}$ & 13,5 & \pm & 1,2 & 13,4 & \pm & 0,4 \\
\hline $\mathrm{P}, \% \mathrm{MS}$ & 6,0 & \pm & 0,7 & 5,8 & \pm & 0,3 \\
\hline Hydroxyprolinurie (mg/24 h) & 462 & \pm & 33 & 437 & \pm & 121 \\
\hline
\end{tabular}

Aucun écart significatif; $1 \mathrm{MS}$ : matière sèche osseuse (les os ayant été vidés de leur moelle). 
du régime coexiste avec une augmentation de l'excrétion urinaire de Ca évoque les effets bien connus des régimes hyperprotéiques (Anonyme, 1980). Toutefois, les 2 régimes étant isoprotéiques, c'est plutôt une différence dans la nature des protéines, et en particulier les apports d'aminoacides soufrés (AAS) (Zemel et al., 1981; Schuette et al., 1982; Van Beresteyn \& Visser, 1983), qui pourrait être en cause. Cependant, le calcul indiquant que les 2 régimes contenaient la même quantité d'AAS, il se pourrait que ceux du régime $P P$ aient été mieux digérés que ceux du régime témoin, ce que laissent supposer les résultats obtenus chez le porc par Walker et al. (1986a et b). Ces auteurs, en comparant la rétention azotée et la digestibilité des AA des protéines issues du soja ou de la caséine, observent des valeurs supérieures, en particulier pour la méthionine, avec l'hydrolysat de caséine ou le caséinate de calcium. Toutefois, on devrait alors s'attendre à une augmentation de l'hydroxyprolinurie comme cela a été observé chez l'homme (Schuette et al., 1981) et chez le rat (Van Beresteyn \& Visser, 1983) recevant des apports croissants de caséine, ce qui n'était pas le cas chez les porcs du présent essai. Par ailleurs, plusieurs résultats, chez l'enfant recevant des laits de remplacement (Anonyme, 1988) mais aussi chez le très jeune porcelet (Miller et al., 1965), montrent au contraire que la caséine, en comparaison avec les protéines de soja, améliore, à niveau protéique égal, la minéralisation osseuse. En revanche, chez le rat nourri avec des régimes contenant, soit des protéines de soja, soit de la caséine, la calciurie augmente parallèlement à l'augmentation de la teneur protéique de l'aliment (Van Beresteyn \& Visser, 1983; Graves \& Wolinsky, 1980) mais si la calciurie est toujours plus élevée avec la caséine, le contenu minéral osseux reste le même (Van Beresteyn \& Visser, 1983) et ce, quel que soit le taux protéique. Si l'on se réfère aux résultats de Hendricks et al. (1970) chez le très jeune porcelet, l'augmentation du taux des protéines, sous forme de soja, a un effet négatif sur la minéralisation osseuse et sur l'absorption de $\mathrm{Ca}$ et $\mathrm{P}$, effet qu'on ne retrouve pas ici, puisque l'absorption de $P$ est supérieure avec le régime témoin lequel contenant $22 \%$ de soja, le régime $P P$ n'en contenait que $14 \%$. Enfin, une revue critique très récente remet largement en cause les liens éventuels entre apports protéiques (nature, quantité) et pertes calciques, urinaires et osseuses (Spencer et al., 1988). Au regard de l'ensemble de ces données, il ne semble pas clairement établi que l'on puisse rapporter la légère diminution de l'absorption du phosphore, ni même la plus forte calciurie, à la nature protéique différente du régime PP.

L'autre explication des différences relevées entre les effets des 2 régimes sur le métabolisme phosphocalcique pourrait provenir des apports de phosphore légèrement différents en quantité mais surtout en nature. Ainsi, les porcs témoins consommaient quotidiennement environ $0,5 \mathrm{~g}$ de plus que ceux du lot PP. L'écart au niveau des quantités absorbées est de 0,8 g/jour, ce qui semble se répercuter au niveau de la phosphaturie, également supérieure de $0,8 \mathrm{~g} / \mathrm{j}$ à celle des porcs du lot PP. Les résultats des bilans de $\mathrm{Ca}$ et $P$ pourraient indiquer que le calcium retenu ait été le facteur limitant : les quantités de $\mathrm{Ca}$ retenues sont identiques pour les deux groupes, ce qui expliquerait que le phosphore absorbé "en trop" par les porcs témoins soit excrété dans l'urine. Cette hyperphosphaturie coexistant avec une réduction de la calciurie s'apparente aux résultats observés chez l'homme (Spencer et al., 1965; Schuette et al., 1981; Zemel \& Linkswiller, 1981) en particulier avec une supplémentation de la ration en phosphate acide, ce qui était 
le cas avec l'utilisation du phosphate monosodique pour le régime témoin. Chez le rat également, calciurie et phosphaturie varient en sens inverse (Howe \& Beacher, 1981a et b) lorsque les apports de phosphore, et donc la quantité de $P$ absorbée, augmentent. Chez le porc, l'absorption du phosphore en valeur absolue $(\mathrm{g} / \mathrm{j})$ mais également relative (digestibilité) tend à augmenter avec les apports de phosphore (Vipperman et al., 1974; Hemmer, 1982) ce qui a pour conséquence d'augmenter la phosphaturie tandis que la calciurie diminue. Enfin, chez de jeunes porcs nourris avec des apports de $P$ et Ca variés, les excrétions urinaires de $\mathrm{Ca}$ et $\mathrm{P}$ varient en sens inverse (Shenkel \& Muller, 1984).

Ainsi, les différences des excrétions urinaires de $\mathrm{Ca}$ et $\mathrm{P}$ entre les 2 lots $\mathrm{T}$ et $P P$ seraient surtout la conséquence d'une meilleure absorption du phosphore chez les témoins. Cette absorption plus forte pourrait provenir, au moins en partie, de la nature des apports de phosphore. En effet, les proportions de phosphore végétal des 2 régimes étant comparables (de l'ordre des $2 / 3$ de $P$ total), la différence de nature des apports porte sur $1 / 3$ de $P$ minéral pour le régime témoin contre $1 / 3$ de $P$ lié au phosphopeptide pour l'autre régime. Le phosphate sodique utilisé, soluble, est généralement considéré comme une excellente source de $P$ chez le porc (Guéguen, 1970; Guéguen \& Rérat, 1965; Cromwell, 1985) ce qui, ajouté au fait que l'apport est légèrement supérieur, expliquerait la plus grande absorption de $\mathrm{P}$ chez les témoins. Le fait que le calcium soit limitant serait dû à ce que la teneur en calcium des régimes (environ $0,8 \%$ ) ait été légèrement inférieure à celle recommandée pour les porcs du même âge $(0,95$ à $1 \%$; Guéguen \& Perez, 1981). Cela est une conséquence de l'ajustement de la parité des apports de $\mathrm{Ca}$ entre les 2 régimes, en raison d'une richesse moindre en $\mathrm{Ca}$ (7 \%) du phosphopeptide que celle initialement prévue $(9 \%)$ et à la volonté de conserver une proportion de $\mathrm{Ca}$ apportée par le phosphopeptide aussi élevée que possible dans le régime PP.

En conclusion, avec des apports $\mathrm{P} / \mathrm{Ca}$ quantitativement très voisins mais qualitativement assez différents, les 2 régimes ont permis des performances comparables et surtout ont conduit à des paramètres osseux pratiquement identiques comme l'étaient les rétentions de $\mathrm{Ca}$ et $\mathrm{P}$ mesurées au cours des bilans. Les résultats ne permettent pas de penser que la nature phosphopeptidique d'une fraction importante des apports $\mathrm{P} / \mathrm{Ca}$ en a amélioré l'utilisation. Cela rejoint les travaux récents portant sur l'utilisation comparée du calcium des produits lactés et de celui des autres sources d'apport, signalés dans l'introduction. II recoupe d'autres observations obtenues récemment (Pointillart \& Drueke, données non publiées) chez des rats pour lesquels, que les apports calciques soient normaux $(0,6 \%)$ ou excessifs $(1,5 \%)$, la résistance à la rupture des fémurs (et leur contenu en cendres) n'était pas influencée par la nature protéique des régimes : $30 \%$ de caséine comparés à $30 \%$ d'isolat de soja. A noter que les seuls effets positifs de la caséine observés in vivo sur la minéralisation osseuse (Hendricks et al., 1970; Anonyme, 1988) l'ont été chez les sujets (porcelet, enfant) en âge de recevoir une alimentation lactée et donc habitués à ce que l'essentiel des minéraux soit sous forme de phosphocaséinate de calcium, ce qui pourrait suffire à en expliquer la meilleure utilisation. II ne nous semble pas que la durée de l'expérience et donc la nécessité d'une adaptation soient ici en cause si l'on se réfère aux modifications des paramètres osseux observées après 6 semaines seulement chez des porcs 2 fois plus 
âgés recevant $20 \%$ de leurs apports $\mathrm{P} / \mathrm{Ca}$ sous forme de yaourt (Pointillart et al., 1986). Enfin, quand on met en parallèle la caséine non plus aux protéines de soja qui, au moins en excès, peuvent avoir des effets rachitigènes chez le porcelet (Miller et al., 1965), mais à la fibrine, cette dernière améliore l'absorption calcique in vitro mais pas in vivo, l'utilisation de $\mathrm{Ca}$ et $\mathrm{P}$ étant alors la même pour les 2 types de protéines lors d'apport calcique normal (Wilson \& Schedl, 1981). Peut-être faut-il penser, comme le suggèrent Srinivasan \& Rao (1979) que les caséinophosphopeptides résistant à la trypsine ne survivent pas assez longtemps dans l'intestin pour influer sur l'absorption calcique.

\section{REMERCIEMENTS}

Nous tenons à remercier M. H. Roy (Atelier de préparation des aliments expérientaux, INRA) pour son aide précieuse dans la fabrication des aliments.

\section{RÉFÉRENCES}

Anonyme (1980) Urinary calcium and dietary protein. Nutr. Rev. 38, 9-10

Anonyme (1988) Bone mineralization and growth in term infants fed soy-based or cow milk-based formula. Nutr. Rev. 46, 152-154

Blake H.H. \& Henning S.J. (1988) Absorption and transport of milk calcium by infant rats. Am. J. Physiol. 254 (Gastrointest. Liver Physiol. 17), G12-19

Cromwell G.L. (1985) Bioavailability of minerals : phosphorus as a model. Proc. Alltech. 1st Ann. Biotechnology Symp. Lexington, Kentucky, 9 pp.
Gerber H.W. \& Jost R. (1986) Casein phosphopeptides : their effect on calcification of in vitro cultured embryonic rat bone. Calcif. Tissue Int. 38, 350-357

Graves K.L. \& Wolinsky I. (1980) Calcium and phosphorus metabolism in pregnant rats ingesting a high protein diet. $J$. Nutr. 110, 24202432

Greger J.L., Krzykowski C.E., Khasen R.R. \& Krashoc C.L. (1987) Mineral utilization by rats fed various commercially available calcium supplements or milk. J. Nutr. 117, 717-724

Guéguen L. (1970) Les critères de qualité nutritionnelle des compléments minéraux en alimentation animale. Bull. Soc. Sci. Hyg. Aliment. 58, 115-129

Guéguen L. \& Perez J.M. (1981) A reevaluation of recommended dietary allowances of calcium and phosphorus for pigs. Proc. Nutr. Soc. 40, 273-278

Guéguen L. \& Rérat A. (1965) Contribution à l'étude de l'absorption intestinale et du mode d'excrétion du phosphore chez le Porc. C.R. Acad. Sci. (Paris) 260, 5112-5115

Hemmer von H. (1982) Der Einfluß der Phosphorversorgung auf die intestinale Absorption von Phosphor bei wachsenden Schweinen. Z. Tierphysiol., Tierernaehr Futtermittelkd. 47, 220-230

Hendricks D.G., Miller E.R., Ullrey D.E., Hoeffer J.A. \& Luecke R.W. (1970) Effect of source and level of protein on mineral utilization by the baby pig. J. Nutr. 100, 235-240

Howe J.C. \& Beecher G.R. (1981a) Effect of dietary protein and phosphorus levels on calcium and phosphorus metabolism of the young fast growing rat. J. Nutr. 111, 708-720

Howe J.C. \& Beecher G.R. (1981b) Effect of dietary protein and phosphorus levels on calcium and phosphorus metabolism of adult rats. Nutr. Rep. Int. 24, 919-929

Lee Y.S., Noguchi T. \& Naito H. (1979) An enhanced intestinal absorption of calcium in the rat directly attributed to dietary casein. Agric. Biol. Chem. 2009-2011

Lee Y.S., Noguchi T. \& Naito H. (1980) Phosphopeptides and soluble calcium in the small intestine of rats given a casein diet. $B r$. J. Nutr. 43, 457-467

Lee Y.S., Noguchi T. \& Naito H. (1983) Intestinal absorption of calcium in rats given 
diets containing casein or aminoacid mixture : the role of casein phosphopeptides. Br. J. Nutr. 49, 67-76

Miller E.R., Ullrey D.E., Zutaut C.L., Hoefer J.A. \& Luecke R.L. (1965) Comparisons of casein and soy proteins upon mineral balance and vitamin D2 requirement of the baby pig. $J$. Nutr. 85, 347-354

Mykkanen H.M. \& Wasserman R.H. (1980) Enhanced absorption of calcium by casein phosphopeptides in rachitic and normal chicks. J. Nutr. 110, 2141-2148

Naito H. \& Suzuki H. (1974) Further evidence for the formation in vivo of phosphopeptide in the intestinal lumen from dietary $\beta$-casein. Agric. Biol. Chem. 38, 1543-1545

Pointillart A., Cayron B. \& Guégen L. (1986) Utilisation du calcium et du phosphore et minéralisation osseuse chez le porc consommant du yaourt. Sci. Aliments 6, 15-30

Recker R.R., Bammi A., Barger-Lux M.J. \& Heaney R.P. (1988) Calcium absorbability from milk products, an imitation milk, and calcium carbonate. Am. J. Clin. Nutr. 47, 93-95

Reeves R.E., Latour N.G. (1958) Calcium phosphate sequestering phosphopeptides from casein. Science 128, 472

Sato R., Noguchi T. \& Naito H. (1983) The necessity for the phosphate portion of casein molecules to enhance $\mathrm{Ca}$ absorption from the small intestine. Agric. Biol. Chem. 47, 24152417

Sato R., Noguchi T. \& Naito H. (1986) Casein phosphopeptide enhances calcium absorption from the ligated segment of rat small intestine. J. Nutr. Sci. Vitaminol. 32, 67-76

Shenkel von H. \& Muller M. (1984) Stoffwechseluntersuchungen an Ferkeln nach unterschiedlicher Calcium- und Phosphorversorgung. Landwirtsch. Forsch. 37, 117-126

Schuette S.A., Hegsted M., Zemel M.B. \& Linkswiller H.M. (1981) Renal acid, urinary cyclic AMP, and hydroxyproline excretion as affected by level of protein, sulfur aminoacid, and phosphorus intake. J. Nutr. 111, 2106-2116 Sheikh M.S., Santa Ana C.A., Nicar M.J., Schiller L.R. \& Fordtran J.S. (1987) Gastrointestinal absorption of calcium from milk and calcium salts. N. Engl. J. Med. 317, 532-536
Smith K.T., Heaney R.P., Flora L. \& Hinders S.M. (1987) Calcium absorption from a new calcium delivery system (CCM). Calcif. Tissue Int. 41, 351-352

Spencer H., Kramer L. \& Osis D. (1988) Do protein and phosphorus cause calcium loss ? J. Nutr. 118, 657-660

Spencer H., Menczel J., Lewin I. \& Samachson J. (1965) Effect of high phosphorus intake on calcium and phosphorus metabolism in man. J. Nutr. 86, 125-132

Srinavasan M.R. \& Rao M.V.L. (1979) Availability of calcium and phosphate in calcium caseinate. J. Food Sci. Technol. (India) 16, 9599

Van Beresteyn E.C.H. \& Visser R.M. (1983) Invloed van soort hoeveelheid eiwit in de voeding op de botontkalking. Zuilvelzicht 12 , 279-281

Vipperman P.E., Peo E.R. \& Cunningham P.J. (1974) Effect of dietary calcium and phosphorus level upon calcium, phosphorus and nitrogen balance in swine. J. Anim. Sci. 38, 758-765

Walker W.R., Maxwell C.V., Owens F.N. \& Buchanan D.S. (1986a) Milk versus soybean protein sources for pigs. I. Effects on performance and digestibility. J. Anim. Sci. 63, 505-512

Walker W.R., Maxwell C.V., Owens F.N. \& Buchanan D.S. (1986b) Milk versus soybean protein sources for pigs. II. Effects on animo acid availability. J. Anim. Sci. 63, 513-524

Wilson H.D. \& Schedl H.P. (1981) Effects of casein and fibrin on calcium absorption and calcium homeostasis in the rat. Dig. Dis. Sci. $26,237-242$

Wong N.P. \& Lacroix D.E. (1980) Biological availability of calcium in dairy products. Nutr. Rep. Int. 21, 673-680

Zemel M.B. \& Linkswiller H.M. (1981) Calcium metabolism in the young adult male as affected by level and form of phosphorus intake and level of calcium intake. J. Nutr. 111, 35-324

Zemel M.B., Schuette S.A., Hegsted M., Linkswiller H.M., (1981) Role of the sulfurcontaining aminoacids in protein-induced hypercalciuria in Men. J. Nutr. 111, 545-552 